\title{
EVALUATION OF TECHNOLOGY IN MODERN SUPPLY CHAIN MANAGEMENT USING BLOCKCHAIN
}

\author{
VAISHNAVI PAWAR ${ }^{1}$, PRIYA YADAV ${ }^{2}$, RUTUJA AWADE ${ }^{3}$, SUMEDHA BHAGWAT ${ }^{4}$, \\ MRS. PREETI PATIL ${ }^{5}$
}

1,2,3,4 UG Students, Dept. Of Information Technology, Dr. D. Y. Patil Institute of Technology, Pimpri, Pune, Maharashtra, India

${ }^{5}$ Asst. Prof. Dept. Of Information Technology, Dr. D. Y. Patil Institute of Technology, Pimpri, Pune, Maharashtra, India

\begin{abstract}
As there is an enormous progress in the Information Technology field, there is also a rise in the economic which in turn creates a competition between the companies. In the Blockchain era we have entered the Supply Chain Management (SCM) System. Compared to the traditional Supply Chain Management System the Supply Chain Management using Blockchain is more effective and useful in its own ways, it also surpasses the limitations of the traditional supply chain management.

Supply chain acts as a primary concern in the business field while transporting of the products among the producer and consumer in the right and systematic way. Many companies across the globe face many challenges to manage the supply chain from source to destiny. We have created a system which maintains a transparency of products and its source in Supply Chain using Blockchain. The objective of this system is the development of an integrated information system in which the digital stream of data generated in the supply chain of products can be assigned to these products. That's the reason why the majority of companies nowadays approach this concept. There are significant advantages of adopting Blockchain in supply chain management, they are: Customer Fulfilment, Anti-Counterfeit, Identify the problems, cost efficiency and rise in Economy.
\end{abstract}

Key Words: Blockchain, Supply chain, source, transparency, products, Counterfeit product, QR code

\section{INTRODUCTION}

Blockchain is a technology which is seen to be effective once adopted in various fields. As in the Fig. 1.1, The pie chart shows the stance of how many countries have adopted Blockchain. As India is a developing country it has noticed an enormous growth in the Technology field. Blockchain Technology's use have been tested in the areas of Trade Finance, Cross-border Payments, Bill Discounting, Supply chain financing, Loyalty and Digital Identity areas. But most of the major fields still lack the use of Blockchain Technology. Some of the Indian banks, business conglomerates, and one stock exchange are among the pioneers for exploring Blockchain in India. In India most of the applications there is still the use of a third party in Supply Chain management (SCM).
In India $46 \%$ of the population is self-employed, they have their own business. Supply chain acts as a primary concern in the business field while transporting of the products among the producer and consumer in the right and systematic way. Many companies across the globe face many challenges to manage the supply chain from source to destiny. Most of the consumers now-a-days are conscious and are mostly aware of what products they are using. They are self-aware and mostly prefer usage of animal and plant cruelty free products. Most of the times companies tend to change the details of the product to increase the sale of the product.

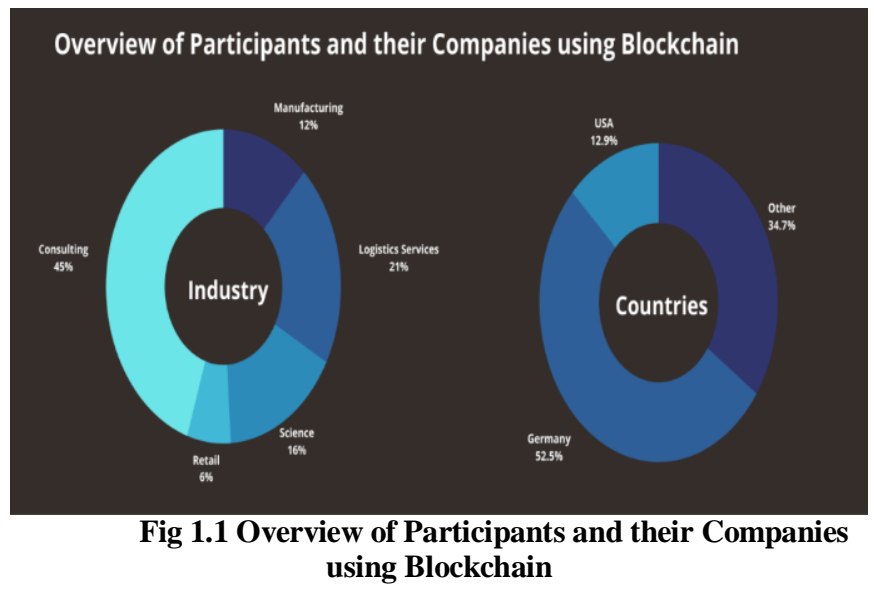

This paper is structured are as follows: Section 1.1 explains the blockchain technology, whereas Problem identification is presented in Section II. Section III gives the problem formulation of the project which includes the concept and objective of the project. Section IV gives a brief survey of Literature done in the research process. Section V gives the working, while Section VI mentions the Research Methodology. Finally, the further Section describes the conclusion and proposes suggestions for further research.

\subsection{Introduction to Blockchain}

Blockchain is a new type of data system that maintains and records all the details regarding to the field in a way that permits multiple stakeholders to confidently share access to the same data and information.

A Blockchain is a type of Distributed ledger technology (DLT), which means it is a data ledger that is shared by multiple entities operating on a distributed network. Fig 1.2 shows how a transaction gets into the Blockchain. When a 
transaction is requested and authenticated through the platform a new block is created. The Blockchain technology operates by recording and storing every transaction across the network in a cryptographically linked block structure that is replicated across network participants. The block which is created regarding the transaction is sent to every node in the network. Nodes validate the transaction. Typically, in Cryptocurrency it is noticed that nodes i.e., participants receive a reward for proof of work. Each block in the blockchain has a hash, which is the output of an algorithm that turns the contents of the block into a random mix of letters and numbers.

By mathematically validating that the hashes match the expected values, users can trust that the data has not been tampered with. Relevant actors or organizations participating in the distributed network can serve as "nodes" to participate in consensus, a process that keeps each blockchain node in sync and handles the addition of new blocks. The block is then added to the existing blockchain and the update regarding it is distributed across the network. Hence the transaction is completed. There are different mechanisms that could be used to establish consensus that have trade-offs between confidentiality, throughput, and security, and vary depending on the implementation and use case.

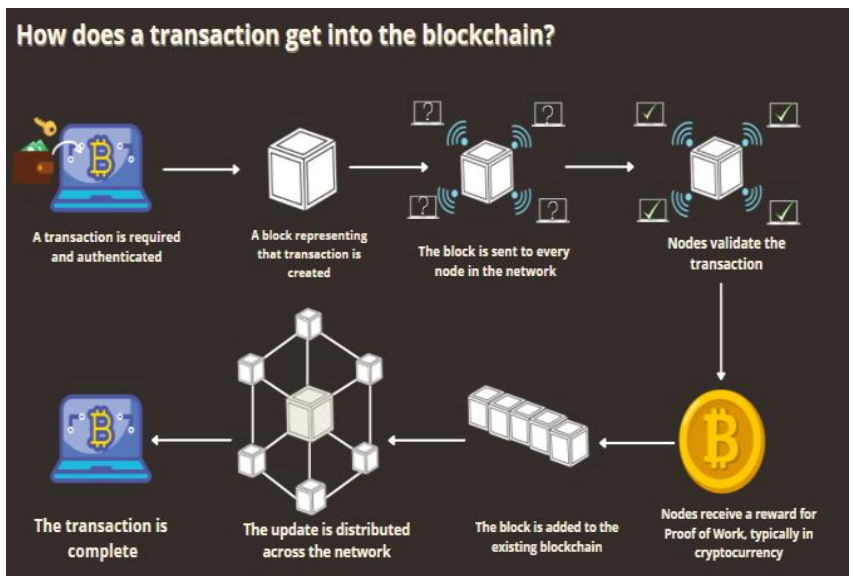

Fig. 1.2 How a transaction works in a Blockchain

There are different types of Blockchains:

- Public Blockchain,

- Private Blockchain

- Consortium Blockchains

- Hybrid blockchains

Depending on the type of blockchain platform being used, blockchains can be designed to provide different levels of access to the data on the blockchain ("on-chain" data). This means that it can provide an increase in transparency of the data between the consumer, while upholding privacy where needed. For instance, a blockchain could enable patients to regulate their own health data and choose who can access their health data.

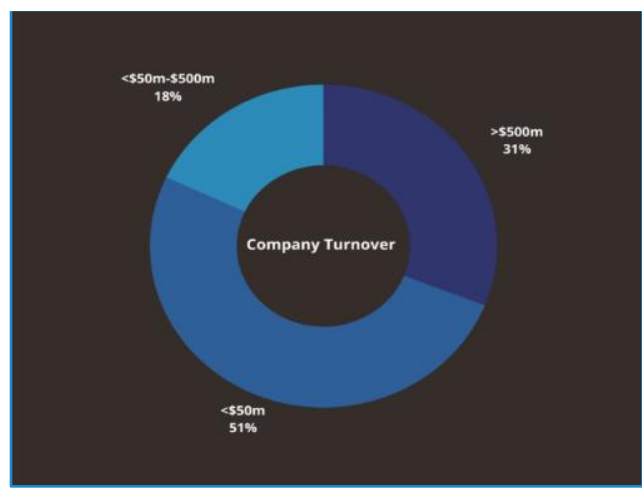

Fig. 1.3 Overview of Turnover achieved by the Companies using Blockchain

Using blockchain during this way establishes trust in the validity of the information and improves the potential to share the info across silos while keeping sensitive data protected. The above Fig.1.3 is a pie chart which gives the overview of the companies turn over using block chain technology. This pie chart shows us that Blockchain is a major contributor in majority of the companies with a turnover of above 550 million dollars. $51 \%$ of the company's turnover who are using the blockchain technology have experienced a turnover of above 550 million. $31 \%$ companies have experienced a turnover below 550 million dollars whereas $18 \%$ companies have experienced a turnover between 50 million to 500 million dollars.

\section{PROBLEM IDENTIFICATION}

Due to the rapidly changing industries and economy, there is a need to identify the problems that lack in the traditional SCM. As most of the problems regarding the growth of the company selling their goods lies at the start of the product formation. The most primary concern regarding it is the supply chain. Most of the expenditure of the income of the company is done on getting the raw materials.

To avoid the expenditure cost most companies tend to use low quality raw materials for their product which in turn reduces the quality of the product and violates the trust between the customers and manufacturers.

Thus, it brings to our knowledge that the traditional methods of the SCM are not sufficient and satisfactory for keeping a transparency between the supplier and consumers. Due to these, some major problems are identified and to overcome these problems some ideas and knowledge of a new technology Blockchain is adopted. Following are the problem:

1. Raw materials used are mostly of low quality and consumers are mostly unaware of it.

2. The delay of delivered products between the customers.

3. Do not offer transparency in supply chain as they do not allow the supply chain nodes to verify the authenticity of a product's data.

4. Doesn't fulfill the customers' requirements

5. In available system the data regarding the product can be manipulated and is mostly lost in the process. 
6. Most of the expenditure is done in SCM which turn affects the company's turnover

7. The existing business models include various processes associated with sending, validating, and reconciling data among different parties, where there is no security in the data and the data can't be shared across multiple actors, also there is a need to revalidate and reconciliate data which in terms affect the economy.

8. Most of the products are counterfeit.

\section{PROBLEM FORMULATION}

We are going to design a system which maintains a transparency of products and its source in Supply Chain using Blockchain. Using this proposed method, we are going to implement the two features while delivering the products from producer to consumer. First, we will reduce the delay during the delivery of goods. There will be use of the smart tag's wholesaler/retailer/consumer know the product information and delivery details of the products can collect. Second, we improve the trust level between the consumer and manufacturer.

That is wholesaler/retailer/consumer get the merchandise from producer using the smart tag to understand the detail about particular product (example: Company name, Manufacturing Date, etc.)

\subsection{Concept:}

The motto to introduce the blockchain system in SCM is to increase the transparency between the consumers and manufacturers. To eliminate the third party between the consumer and manufacturers. To reduce the expenditure cost in SCM and to improve and maintain the quality of the product. The concept of the work is,

1. Observe and study the traditional supply chain and to identify the important variables in it.

2. Quantify the important method.

3. Investigate all various fields of management regarding the supply chain.

4. Develop a prototype system which will help in controlling the overall process.

5. Produce a specification which will develop a system that will help in reduction of expenditure.

6. Refined design of the SCM using Blockchain and implement the project.

Considering the above concept points we will design a system using Blockchain which helps in replacing the traditional Supply chain.

\subsection{Objective:}

The objectives of this project are:

- The objective is to develop an integrated information system in which the digital stream of data generated in the supply chain of products can be assigned to these products.

- To Design a system using Blockchain to overcome Counterfeit of products.

- $\quad$ To secure the details of the product using a QR code.

- $\quad$ Provide security and trust between the customer and manufacturer by offering data to customer.

- No manipulation of the stored data can be done.

- Furthermore, the information should enable traceability of product, its sub-products and all steps in manufacturing with their associated quality parameters

\section{LITERATURE SURVEY}

\subsection{Introduction:}

Here we present a review on tracing the source of the product in the supply chain. Due to the traditional supply chain Counterfeit products are growing exponentially with the enormous amount of online and black-market. So, there is a strong need to address the challenges of detecting the source of the products and designing appropriate technology to improve detection accuracy. It is one of the active research areas to be explored in the current world.

Research Papers:

1. Design of an efficient image protection method based on QR code (IEEE-2021): In this paper, the author proposed a technique to protect image data based on QR codes and describe the design and verification results. In the aspect of image protection, the technology presented in this paper can enhance security through the creation of a secure image with both de-identification and encryption applied. In terms of QR code, our proposed method optimizes data to meet capacity limits and to eliminate security vulnerabilities.

2. A Blockchain-Based Application System for Product Anti-Counterfeiting (IEEE-2021): This paper is the first Blockchain system that proposes a fully functional anti-product forgery system. By paying a very low transaction fee, users of our system no longer need to be concerned about the possibility of acquiring a counterfeited product.

3. Fake Product Detection Using Blockchain Technology (IEEE-2021): In this paper, the author proposed a system which is useful for the customer to detect fake products in the supply chain. Customers are able to scan QR codes which is assigned to the product and can get all the information like transaction history, current owner based on which end-user can check whether the products they bought are authentic or not.

4. An Efficient Bar/QR Code Recognition System for Consumer Service Applications (IEEE-2020): This paper presents an image processing-based method for automatic analysis of Bar/QR codes. The proposed method involves the filtering of the images which 
removes the noises associated in the images. Further, the images are converted into grey scale and binarization is performed. The experiments conducted using this yielded $87 \%$ accuracy

5. A Blockchain-based Supply Chain Quality Management Framework (IEEE-2020): In this paper, the author proposed a blockchain-based framework. The framework comes up with a theoretical basis for brilliant quality management of the supply chain which is based on the Blockchain Technology. Furthermore, it imparts a foundation to develop theories related to data resource management in distributed, virtual organizations.

6. Smart Tags for Brand protection and anticounterfeiting in the wine industry (IEEE-2020): This paper describes a brand protection and anticounterfeiting solution for the wine industry based on smart tags and Cloud enabled technologies. The main proposal utilized in this technique are the usage of smart tags which are used to utilize quick response codes and functional inks supported by the Cloud system and which maintains a two-way communication between the winemaker and the enduser.

\subsection{Company's view on Blockchain:}

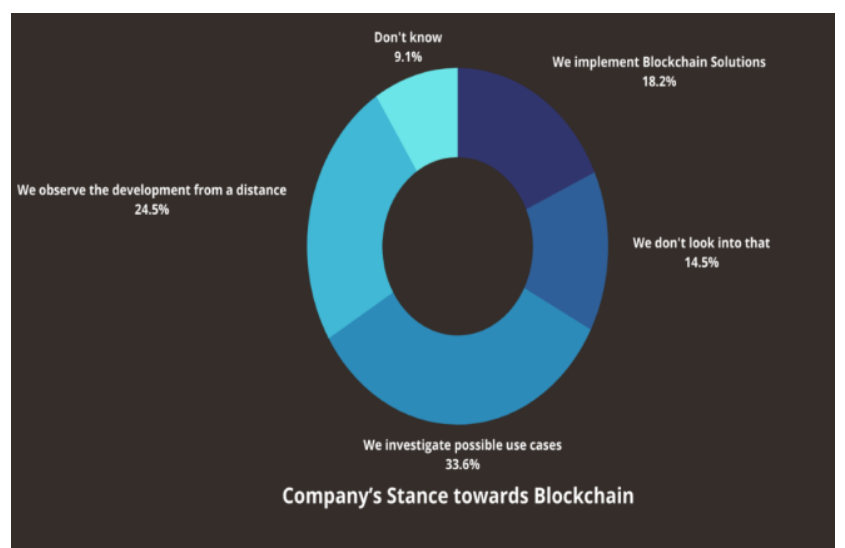

Fig. 4.2.1 Overview of Turnover achieved by the Companies using Blockchain

The above pie chart gives us an idea of the company stance towards blockchain technology. Around 24.5\% companies observe the development from a distance and do not use the technology. $33.6 \%$ companies investigate the possible use cases. $14.5 \%$ companies are not interested in the technology. While $18.2 \%$ have successfully implemented the blockchain technology.

\section{WORKING PRINCIPLE}

As noticed in the previous section and in the Fig. 5.1 there is no access to data related to the product to the customers. With blockchain data can be accessed and verified by all the actors present in the system, rather than merely by the original certifier. Blockchain removes the need for a trusted central organization that operates and maintains the supply chain system. Using Blockchain in the system as a shared and a perfect secure platform, we would be able to create a system where all data is safe and distributed, where even consumers will have an access.

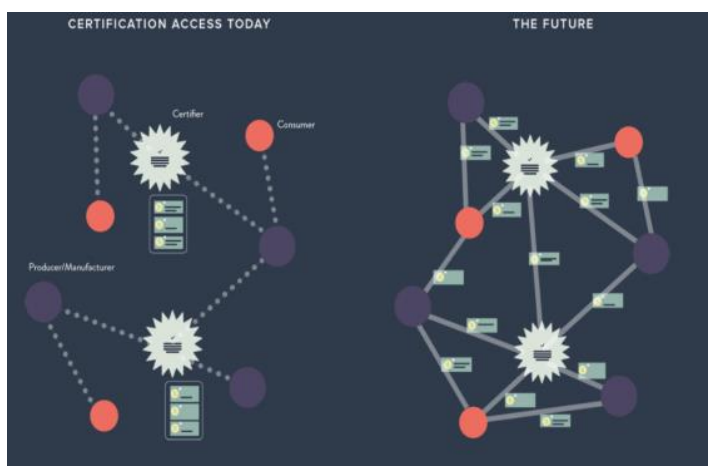

Fig. 5.1 Working of SCM Database Traditional (a) \& Blockchain (b)

Most of the existing certification and the access to the data in the supply chain can pave a new path implemented on the blockchain system. There are six different types of actors involved in working as shown in the Fig. 5.2:

1. Producers i.e., Raw materials are collected from the producers.

2. Manufacturers, they convert the raw materials to products.

3. Distributers, they are the standard organizations that provide credentials and a unique identity to actors, they also define the rules of certain scheme in the system.

4. Retailers, also known as Certifiers and auditors, they act separate agents to maximize the inspection between producers and manufacturers and verify annual turnover.

5. Consumers i.e., Customers, the buyers of products all along a supply chain, including the end consumer.

6. Purchase is the last step in the supply chain were the consumers buy and the products are delivered to them.

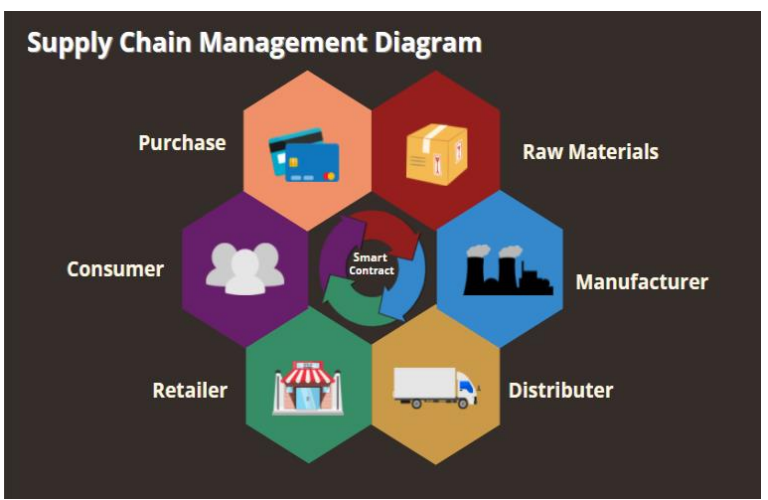

Fig. 5.2 Supply Chain Management Diagram

In the Fig. 5.3 and 5.4 the top row of blocks represents web interfaces and applications, the bottom layer(s) are data handling and storage. Today your data in the system is stored 
in a silo. With blockchains your data are going to be stored publically or pseudo anonymously in the shared database. This diagram is adapted from the Provenance paper on Blockchain for better understanding of the study, the diagram is a concept of Nick Grossman, GM at Union Square ventures, however his also includes time.

The current traditional Data layer in the system shown in Fig. 5.3 there is no customer interface. The data handling and the storage can be only accessed by certifier interface and Producer/Manufacturer interface. There is a surge in attacks done on the centralized traditional systems which in turn affects in data manipulation and data loss. By leveraging those with the most elevated access rights, an attack can target IT and operational support administrators could eventually lead to the system being fully compromised.

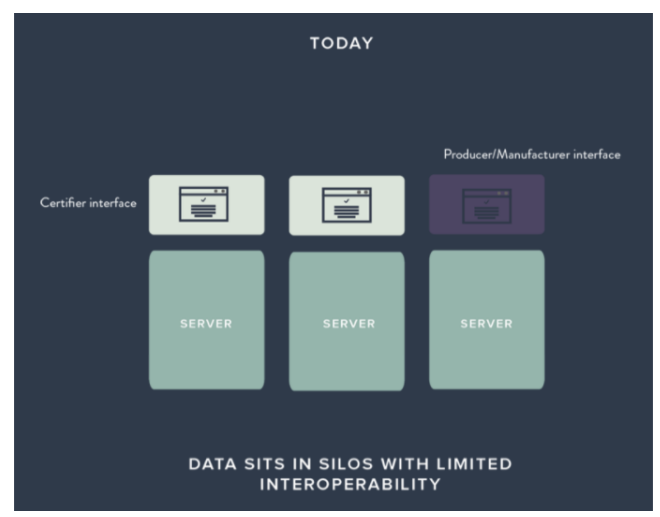

Fig. 5.3 Data Layer in Traditional SCM

Blockchain plays a big role in security, it does not matter who or where the user is situated, because the data provided to the system in Blockchain is only accepted only if it is authenticated. By using Blockchain, we can create a system that allows in adoption of new model, which solves all the problems identified from the traditional system. Blockchain works like a data system, it takes input and carries out actions based on these inputs, modifying the database in a perfect orderly manner which is determined according the program.

The magic of the blockchain and its surrounding incentive structure is such that the users can unambiguously discover the state of the system, not from a single particular authority but by independently applying common rules and issuing data openly.

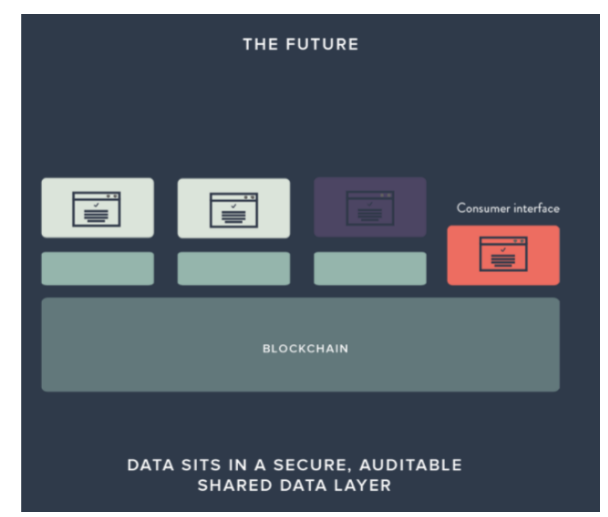

Fig. 5.4 Data Layer in Blockchain SCM

The Blockchain system in Fig 5.4 shows the system representation in the front end and back end. There is a new interface included in the system, it is the consumer interface. This system alone develops the fundamental trust relationship between the consumer and the system as a whole. This helps in maintaining transparency between the consumer and manufacturer. It solves most of the problems identified in the previous SCM.

The system will initially be deployed by the admin, who implements a process for the registration of named participants (i.e., certifiers, auditors, producers, and manufacturers). Such participants may request enrollment of their digital identity, which links their real-world identity with their blockchain-based digital identity, thus permitting them to interact with the blockchain using their real-world identity. Upon request, the registration authority verifies their identity and records the outcome in the blockchain, available for all to examine.

\section{RESEARCH METHODOLOGY}

The main research done in any scientific field is to impart a conclusion which is useful in light of objective of the study done. In order to obtain the significant conclusion, it is essential for the researchers to adopt a suitable method and procedure and explaining it to fulfill the objective of the study. This section dives the detail report of the project.

As seen in the previous sections, the traditional SCM does not provide a transparency between the consumer and manufacture. Most of the products are now-a-days provided with the QR tag but the permission to access the details in it are not given to the consumer. This problem will be solved by using a blockchain network in the supply chain, as it crosses overs the gap between the database. Now there will be only one database which is the Blockchain distributed layer, where the consumers will have the same access to the products detail as the manufacturer. Issues like low quality raw materials used, exceeded expenditure of the income on the raw materials and forging of the goods will be satisfied to a great extent by reducing expenditure of income on the SCM and arranging a proper system where from the process of manufacturing to the delivery of the product will be done in a genuine manner.

As Blockchain Supply Chain Management is opted, there will be transparency in supply chain as they will allow the supply chain nodes to verify the authenticity of a product's data. So even if there will be use of low quality or harmful products used during the production the consumers will be aware of it. Data plays a crucial role in today's business world. In the traditional SCM the data regarding the product is manipulated and lost in the process so to overcome it a secure encryption and verification technologies are used which are present in Blockchain. This ensures that no data is manipulated and lost. Research methodology deals with design and implementation of all the components which are used in the system with required modification. Firstly, synthesis regarding all the identified problems which is related to the project is to be done. After the complete overview, the design of the system and then regarding implementation is to be done in the Supply Chain. Parameters of the system will be selected according to the objectives. The project requirements are to be mentioned. System diagram, various Unified Modelling Language (uml) diagram are to be created with labelling. 


\section{CONCLUSIONS}

In this work, we proposed and discussed the adoption of blockchain technology in the supply chain management system. The evolution of blockchain and its application in supply chain businesses is presented which enables the creation of shared, secure, decentralized ledgers, smart contracts, in addition it reduces the use of intermediates which reduce the cost through transactions and operations which is related to supply chain. Using a blockchain-based system could improve the speed at which data can be collected from diverse locations. To embrace users to adopt a blockchain system, the system needs to provide value. Understanding the full implications of blockchain technology in the supply chain management requires multidisciplinary implications and the involvement of organizations to develop standards and rules on blockchain implementation. Beyond, there is a substantial amount of work in this area for future research direction.

\section{FUTURE SCOPE}

We believe that eventually global trade will prosper into a battle of supply chains, where efficiency, block chain optimization, and innovative financing will yield best in breed organizations. As blockchain obtain momentum, "there are going to be real-time visibility, reduced complexities, improved accuracy and efficiency within the system, which successively will help reduce costs and enhance trust. Its usefulness won't be limited to shippers or customers but will reach lowering trade barriers, especially for developing nations which aren't ready to participate in global trade owing to cost considerations".

Also, blockchain can be integrated in the Internet of Effects device operation, advancing confidence, and P2P insurance. All of these operations of blockchain are formerly being vetted and show favorable results.

\section{REFERENCES}

[1] Young-Sae Kim, Jin-Hee Han, Geon-woo Kim, "Design of an efficient image protection method based on QR code (IEEE2021)"

[2] Kentaroh Toyoda, Takis Mathiopoulos, "A Blockchain-Based Application System for Product Anti-Counterfeiting (IEEE2021)".

[3] Tejaswini Tambe, Sonali Chitalkar, Manali Khurud, Madhavi Varpe, S. Y. Raut, "Fake Product Detection Using Blockchain Technology (IEEE-2021)".

[4] Arju Aman, Aryan Singh, Ayush Raj and Sandeep Raj, "An Efficient Bar/QR Code Recognition System for Consumer Service Applications (IEEE-2020)".

[5] Si Chen, Rui Shi, Jiaqi Yan, Yani Shi, Jinyu Zhang Zhuangyu Ren, "A Blockchain-based Supply Chain Quality Management Framework (IEEE-2020)".

[6] Sanja Radonjic, Stevan Sandi, Jovana Drobnjak, Marko Simeunovic, Biljana Stamatovic, Tomo Povovic, "Smart Tags for Brand protection and anti-counterfeiting in the wine industry (IEEE-2020)".

[7] Sepide Tavassoli, Maryam Sardashti, Naghme Khajeh Nasir Toussi, "Supply Chain Management and Information Technology Support (IEEE-2019)".
[8] Shivendra, Dr.Kasa Chiranjeevi, Dr. Dhananjay D.Maktedar, Mukesh Kumar Tripathi, "Block chain Technology in Agriculture Product Supply Chain (IEEE-2021)".

[9] Manal Hader, Abderrahman Elmhamedi, Abdellah Abouabdellah, "Blockchain technology in supply chain management and loyalty programs: toward blockchain implementation in retail market (IEEE-2020)".

[10] Affaf Shahid, Ahmad Almogren, Nadeem Javaid, Fahad Ahmad AL-Zahrani, Mansour Zuair, Masoom Alam, "Blockchain-Based Agri-Food Supply Chain: A Complete Solution (IEEE-2020)".

[11] Su Lei, Wang Haiying, "Supply Chain Finance Research in Digital Bulk Commodities Service Platform Based on Blockchain (IEEE-2020)".

[12] S. Madumidha, P. S. Ranjani, S. S. Varsinee and P. S. Sundari, "Transparency and Traceability: In Food Supply Chain System using Blockchain Technology with Internet of Things," 2019 3rd International Conference on Trends in Electronics and Informatics (ICOEI), 2019, pp. 983-987, doi: 10.1109/ICOEI.2019.8862726

[13] Provenance, 04 2019, [online] Available: https://www.provenance.org.

[14] R. G.S. and M. Dakshayini, "Block-chain Implementation of Letter of Credit based Trading system in Supply Chain Domain," 2020 International Conference on Mainstreaming Block Chain Implementation (ICOMBI), 2020, pp. 1-5, doi: 10.23919/ICOMBI48604.2020.9203485.

[15]. S. NASIH, S. AREZKI and T. GADI, "Enhancement of supply chain management by integrating Blockchain technology," 2019 1st International Conference on Smart Systems and Data Science (ICSSD), 2019, pp. 1-2, doi: 10.1109/ICSSD47982.2019.9002771.

[16]. J. C. López-Pimentel, O. Rojas and R. Monroy, "Blockchain and off-chain: A Solution for Audit Issues in Supply Chain Systems," 2020 IEEE International Conference on Blockchain (Blockchain), 2020, pp. 126-133, doi: 10.1109/Blockchain50366.2020.00023.

[17] F. Qu, H. Haddad and H. Shahriar, "Smart Contract-Based Secured Business-to-Consumer Supply Chain Systems," 2019 IEEE International Conference on Blockchain (Blockchain), 2019, pp. 580-585, doi: 10.1109/Blockchain.2019.00084

[18] R. Kumar and R. Tripathi, "Traceability of counterfeit medicine supply chain through Blockchain," 2019 11th International Conference on Communication Systems \& Networks (COMSNETS), 2019, pp. 568-570, doi: 10.1109/COMSNETS.2019.8711418 